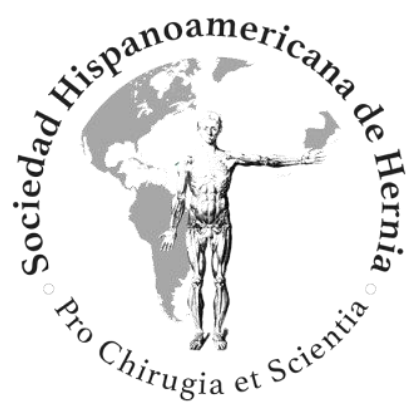

Hernias incisionales laterales: un desafío quirúrgico

\title{
Lateral incisional hernia: surgical challenge
}




\section{Hernias incisionales laterales: un desafío quirúrgico}

Lateral incisional hernia: surgical challenge

Camilo Ramírez-Giraldoㅁ, Viviana Orozco-Martín ${ }^{1}$, Carlos Rey-Chaves ${ }^{2}$, Andrés Isaza-Restrepo ${ }^{1}$

${ }^{1}$ Servicio de Cirugía General. Hospital Universitario Mayor Méderi. Universidad del Rosario. Bogotá (Colombia). ${ }^{2}$ Hospital Universitario Mayor. Bogotá (Colombia)

Autor para correspondencia: Camilo Ramírez Giraldo. Universidad del Rosario. Cl. 14, \#625. Bogotá (Colombia).

Correo electrónico: ramirezgiraldocamilo@gmail.com

Recibido: 07-01-2020

Aceptado: 29-01-2020

\section{RESUMEN}

Introducción: Las hernias incisionales laterales han sido poco estudiadas. Su manejo quirúrgico es un reto debido a las prominencias óseas y a las estructuras neurovasculares, lo que dificulta la fijación de la malla.

Objetivo: El objetivo de este estudio es describir nuestra serie de hernias laterales con el fin de mostrar la experiencia en cuanto al tipo de malla, el abordaje quirúrgico y la recidiva y así ampliar el conocimiento sobre esta patología cada vez más importante.

Métodos: Estudio descriptivo de una serie de pacientes con hernias incisionales laterales reparadas quirúrgicamente entre febrero de 2015 y noviembre de 2018 en un hospital de alta complejidad.

Resultados: Entre 2015 y 2018, 27 pacientes, con una mediana de edad de 70 años (RQ: 12 años) y una mediana de índice de masa corporal de $27.9 \mathrm{~kg} / \mathrm{m}^{2}$ (RQ: $5.36 \mathrm{~kg} / \mathrm{m}^{2}$ ), ingresaron para ser 
intervenidos de hernias laterales. Se realizó un seguimiento con una mediana de 14 meses (RQ: 4.2), con evidencia de tres recidivas durante el seguimiento. La mayoría de las hernias se localizó en el flanco (L2) (48.1\%). La apendicetomía (29.6 \%) fue el antecedente quirúrgico más frecuente. La técnica quirúrgica más comúnmente empleada fue una malla premuscular (51.8 \%), con uso de mallas de polipropileno predominante $(81.4 \%)$.

Conclusiones: Actualmente no contamos con una técnica para el manejo estandarizado de los defectos laterales de la pared abdominal, por lo que se requieren estudios controlados aleatorizados que comparen las distintas técnicas para definir la más segura y con menor tasa de recidivas.

\section{ABSTRACT}

Introduction: Incisional lateral hernia has not been studied properly, the surgical management is a challenge, because bone and neurovascular structures difficult the mesh fixation.

Objective: The first objective of this study is to describe the cases of lateral incisional hernia, to show the experience in terms of type of mesh, surgical approach, recurrence, and expand the knowledge of this pathology that every day becomes more important.

Methods: A case series of patients with lateral incisional hernia, which undergo surgical repair, between February 2015, and November 2018, in a 4th level hospital in Bogotá, Colombia.

Results: Between 2015 and 2018, 27 patients, with a mean age of 70 years (IQR: 12 years), and a mean of body mass index of $27-9 \mathrm{~kg} / \mathrm{m}^{2}$ (IQR: $5.46 \mathrm{~kg} / \mathrm{m}^{2}$ ), that undergo surgical repair of lateral hernia. We follow patients with a mean of 14 months (IQR: 4.2), with an evidence of recurrence of 3 patients. The majority of the hernias were in flank (L2) (48.2\%) localization. Appendectomy with $29.6 \%$, were the surgical 
procedure with most of the cases. Surgical technique more commonly used was on lay mesh fixation, with a polypropylene mesh material in most of the cases (81.4\%).

Conclusion: Actually we do not have a standardized technique for these defects, for that reason more randomized controlled studies are required comparing the techniques to define a secure approach, with a less rate of recurrence.

Palabras clave: Hernia incisional, herniorrafía, malla, recurrencia.

Keywords: Incisional hernia, hernia repair, surgical mesh, recurrence.

\section{INTRODUCCIÓN}

Las hernias incisionales laterales han sido poco estudiadas. Se han descrito principalmente después de nefrectomías y en reparaciones de aneurismas de aorta abdominal, aunque también se han descrito tras apendicetomías, cierre de estomas y colecistectomías, entre otros procedimientos $^{1-4}$. El manejo quirúrgico es un reto debido a las prominencias óseas y a las estructuras neurovasculares, lo que dificulta la fijación de la malla ${ }^{1}$.

La Sociedad Europea de la Hernia definió en el año 2009 las hernias laterales como las que se encuentran localizadas laterales a los músculos rectos abdominales, inferiores al último arco costal y superiores a la región inguinal. En esta región delimitada las han dividido en hernias del flanco (L2), hernias subcostales (L1), hernias ilíacas (L3) y hernias lumbares (L4), que, además, según el ancho, se han clasificado en menores de $4 \mathrm{~cm}$ (W1), entre 4 y $10 \mathrm{~cm}$ (W2) y mayores de $10 \mathrm{~cm}$ $(\mathrm{W} 3)^{5}$.

Este tipo de patología es un problema que va ganando relevancia a medida que aumenta el número de procedimientos abdominales, como ha sido en el caso de las nefrectomías durante la última década ${ }^{6}$. Su 
frecuencia entre los defectos incisionales de la pared abdominal corresponde hasta el $25 \%$ en centros especializados ${ }^{7}$. Asimismo, debe enfatizarse que la literatura al respecto es escasa. Se ha encontrado una revisión de la literatura en la que se revisaron 15 manuscritos con 1061 pacientes a los que se les realizó una incisión en el flanco y con la presencia de hernia incisional o bulto en 182 pacientes para una incidencia del $17.1 \%$ (1). Es importante destacar que en muchos de estos estudios no se diferencia entre hernia o bulto. El bulto se presenta por una debilidad y atrofia de la pared secundarias al daño nervioso durante la incisión, pero sin defecto aponeurótico. Su incidencia está descrita entre el $19-57 \%{ }^{6}$.

La corrección quirúrgica de este tipo de hernias no se encuentra estandarizada. El objetivo de este estudio es describir nuestra experiencia en hernias laterales (L1, L2 y L3) con el fin de mostrar el tipo empleado de malla y el tipo de abordaje quirúrgico, así como cuantificar las recidivas y ampliar de teste modo el conocimiento sobre esta patología cada vez más importante.

\section{MATERIALES Y MÉTODOS}

\section{Diseño del estudio}

Serie retrospectiva de pacientes con hernias incisionales laterales reparadas quirúrgicamente entre febrero de 2015 y noviembre de 2018, de forma consecutiva, en un hospital de alta complejidad. Se revisaron las historias clínicas para conocer las características sociodemográficas, de imagen y quirúrgicas, así como las complicaciones de estos pacientes. Se realiza una estadística descriptiva utilizando un enfoque cuantitativo para describir las proporciones de las variables de interés.

\section{Técnica quirúrgica}

El procedimiento en todos los casos de hernias de tamaño moderado (no W1) y el procedimiento laparoscópico se realizaron bajo anestesia 
general. En los casos de hernias W1 no laparoscópicas, el procedimiento fue con anestesia regional. Siempre que fue posible hubo cierre primario del defecto. Se realizó resección de la cicatriz y una disección para tener en todos los casos un espacio para solapamiento de la malla con el defecto herniario de por lo menos $5 \mathrm{~cm}$. La localización y el tipo de la malla fue una decisión discrecional por parte del cirujano.

\section{Seguimiento}

Se revisaron las historias clínicas de la consulta ambulatoria. Se tuvo un control a la semana, al mes, a los seis meses, a los doce meses y continuo anualmente. Durante esta consulta se evaluó recidiva al examen físico, además de la presencia de alguna complicación, como seroma, hematoma o infección de la herida quirúrgica.

\section{RESULTADOS}

Un total de 27 pacientes con defectos laterales incisionales de la pared del abdomen fueron sometidos a reparación del defecto herniario en el periodo de tiempo estudiado, de las que 15 eran mujeres (55.6\%). La mediana de edad fue de 70 años (rango intercuartílico [RQ]: 12 años). Las comorbilidades se presentan en la tabla I. Hubo antecedente de tabaquismo en cinco pacientes $(18.5 \%)$ y solo uno refirió haber consumido tabaco tres meses antes de la cirugía. La mediana del índice de masa corporal (IMC) fue de $27.9 \mathrm{~kg} / \mathrm{m}^{2}$ (RQ: $5.36 \mathrm{~kg} / \mathrm{m}^{2}$ ).

Las características de la hernia y del procedimiento quirúrgico, así como la recuerrecia y las complicaciones, se muestran en la tabla II. A 20 pacientes se les realizó una tomografía computarizada de abdomen para planeamiento quirúrgico. No fue solicitada en siete pacientes: los 6 de urgencias y 1 por ser de tamaño W1. En 19 pacientes el procedimiento fue electivo (70.3\%) y en 6 fue un procedimiento de urgencia por obstrucción intestinal o estrangulación $(22.2 \%) .3$ pacientes fueron intervenidos por laparoscopia (11.1\%); el resto, mediante abordaje 
abierto, y la malla fue colocada en el plano premuscular en 14 pacientes (51.8 \%) y en 10 , en el plano retromuscular (37 \%).

La mediana de estancia hospitalaria fue de 4 días (RQ: 4 días). No se presentó ninguna mortalidad en la serie. En 6 pacientes se presentaron complicaciones posoperatorias en los primeros 30 días: 2 íleo posoperatorios, 2 infecciones urinarias asociadas al cuidado de la salud, 1 infección del sitio operatorio superficial y 1 perforación intestinal inadvertida que requirió reintervención.

La mediana del seguimiento fue de 14 meses (RQ: 4.2 meses). El seguimiento únicamente se realizó con valoración en el examen físico, y se encontraron 3 pacientes con recidiva durante este seguimiento: $1 \mathrm{sin}$ malla y 2 con malla en localización retromuscular.

\section{DISCUSIÓN}

Las hernias laterales son un desafío para el cirujano. Su presencia se ha descrito después de apendicetomías, colecistectomías con abordaje subcostal, cierre de estomas y nefrectomías ${ }^{1-4}$. En nuestra serie, solo 5 pacientes no presentaron ninguno de los factores de riesgo descritos en la literatura para la presencia de hernia incisional, como son: diabetes mellitus, hipertensión arterial, enfermedad pulmonar obstructiva crónica, enfermedad coronaria, enfermedad renal crónica de estadio terminal, malignidad, prolapso del estoma o hernia paraestomal. Otros factores de riesgo descritos son el IMC elevado (la mediana en nuestra serie se encuentra en rangos de sobrepeso) y el uso de tabaco, otro factor de riesgo que se encontró en 5 pacientes $(1,4,8)$.

La indicación del procedimiento quirúrgico en todos los casos se debió a que la hernia lateral era sintomática. La presencia de masa o de bulto, seguido del dolor, fueron los más frecuentes y, en estos casos, se realizó un procedimiento electivo. Por el contrario, en 6 casos fue necesario realizar una cirugía de urgencias debido a la sospecha de incarceración. 
En la literatura está descrito que en los pacientes asintomáticos o mínimamente sintomáticos puede seguirse un manejo no operatorio ${ }^{1}$.

La recomendación del planeamiento prequirúrgico con tomografía computarizada de abdomen se siguió en los casos de cirugía electiva o de tamaño moderado (no W1). En los pacientes a los que se les realizo el procedimiento programado se siguieron las recomendaciones de reducción de peso en los obesos, corrección de hiperglicemia, optimización cardiopulmonar y abandono del tabaco como mínimo cuatro semanas antes de la intervención ${ }^{1,9}$.

En cuanto a la técnica quirúrgica, todavía no disponemos de un consenso amplio en la literatura. Hay que tener en cuenta dos consideraciones: el abordaje quirúrgico y el plano de localización de la malla10,11. El abordaje laparoscópico se considera hoy día como una opción aceptada, con tasas similares de recidiva y de morbilidad a las de los de corrección de defectos herniarios de la línea media, aunque nosotros lo utilizamos de forma muy selectiva ${ }^{12-14}$. En el abordaje abierto, lo fundamental es el plano de posicionamiento de la malla, aspecto todavía controvertido en la literatura. Se utiliza más ampliamente y está más aceptada la localización retromuscular ${ }^{1,11,15,16}$. Más importante es tener en cuenta que la malla debe solapar al menos 5-10 cm más allá del defecto herniario, además de estar anclada a estructuras músculoaponeuróticas 0 , de no ser posible, a las prominencias óseas ${ }^{1,10,16}$.

En la literatura, la mayor serie publicada de hernias incisionales laterales ha sido documentada por Moreno-Egea. En su experiencia, se demuestra que los defectos laterales pueden ser intervenidos mediante una técnica de doble reparación protésica con una baja morbilidad y una tasa de recurrencias 0 a 10 años ${ }^{17}$.

Tampoco existe un consenso sobre el tipo de malla que debe usarse. Están descritas el polipropileno, el poliéster y el politetrafluoroetileno, así como mixtas o biológicas, pero existe una recomendación de usar mallas de peso mediano porque tienen menos complicaciones ${ }^{9}$. Lo que sí 
parece evidente es que siempre que sea posible debe usarse una malla en las hernias incisionales. En esta serie, en tres casos no se usó malla por elección personal del cirujano.

En las recidivas es importante subrayar que la ausencia de malla y la presencia de obesidad son los factores relacionados con el desarrollo de este tipo de hernias y de las recidivas en nuestra experiencia. En la literatura también se mencionan como causas de recurrencia el insuficiente solapamiento, el retiro de la malla por infección, la fractura de la malla o una fijación inadecuada ${ }^{1}$.

\section{CONCLUSIÓN}

La corrección de las hernias incisionales laterales sigue siendo un desafío para el cirujano general. Actualmente no disponemos todavía de una técnica de manejo estandarizada; sin embargo, la técnica de doble reparación protésica parece tener resultados adecuados en términos de morbimortalidad, aunque se requieren estudios controlados y aleatorizados que comparen los distintas procesos para definir una técnica segura y con la menor tasa posible de recidivas.

\section{BIBLIOGRAFÍA}

1. Zhou DJ, Carlson MA. Incidence, etiology, management, and outcomes of flank hernia: review of published data. Hernia. 2018;22(2):353-61. DOI: 10.1007/s10029-018-1740-1

2. Peres MA de O, Aguiar HR, Andreollo NA. Surgical treatment of subcostal incisional hernia with polypropylene mesh-analysis of late results. Rev Col Bras Cir. 2014;41(2):82-6. DOI: 10.1590/S0100-69912014000200002 
3. Duce $A M$, Lozano $O$, Villeta $R$, et al. Incisional hernia following appendectomy. Surgical experience. Hernia. 1998;2:169-71. DOI: 10.1007/BF01569140

4. Amelung FJ, de Guerre LEVM, Consten ECJ, et al. Incidence of and risk factors for stoma-site incisional herniation after reversal. BJS Open. 2018;2(3):128-34. DOI: 10.1002/bjs5.48

5. Muysoms FE, Miserez M, Berrevoet $F$, et al. Classification of primary and incisional abdominal wall hernias. Hernia. 2009;13(4):407-14. DOI: 10.1007/s10029-009-0518-x

6. Inkiläinen A, Styrke J, Ljungberg B, et al. Occurrence of abdominal bulging and hernia after open partial nephrectomy: a retrospective cohort study. Scand J Urol. 2018;52(1):54-8. DOI: 10.1080/21681805.2017.1376352

7. Moreno-Egea A, Guzmán P, Morales G, et al. Tratamiento de la eventración no medial: experiencia de una unidad de pared abdominal y revisión de la literatura. Cir Esp. 2007;81(6):330-4. DOI: 10.1016/S0009-739X(07)71332-5

8. Itatsu K, Yokoyama Y, Sugawara G, et al. Incidence of and risk factors for incisional hernia after abdominal surgery. Br J Surg. 2014;101(11):1439-47. DOI: 10.1002/bjs.9600

9. Liang MK, Holihan JL, Itani K, et al. Ventral Hernia Management. Ann Surg. 2017;265(1):80-9. DOI: 10.1097/SLA.0000000000001701

10. Stumpf $M$, Conze J, Prescher $A$, et al. The lateral incisional hernia: Anatomical considerations for a standardized retromuscular sublay repair. Hernia. 2009;13(3):293-7. DOI: 10.1007/s10029-0090479-0

11. Schrittwieser $R$, Kockerling $F$, Adolf $D$, et al. Small and Laterally Placed Incisional Hernias Can be Safely Managed with an Onlay Repair. World J Surg. 2019;43(8):1921-7. DOI: 10.1007/s00268-019-04980-6 
12. Novitsky YW. Laparoscopic repair of traumatic flank hernias. Hernia. 2017;22(2):363-9. DOI: 10.1007/s10029-017-1707-7

13. Moreno-Egea A, Carrillo A, Aguayo L. Midline versus nonmidline laparoscopic incisional hernioplasty: a comparative study. Surg Endosc. 2008;22:744-9. DOI: 10.1007/s00464-0079480-9

14. Moreno-Egea A, Carrillo-Alcaraz A. Management of nonmidline incisional hernia by the laparoscopic approach: results of a long-term follow-up prospective study. Surg Endosc. 2012;26:106978. DOI: 10.1007/s00464-011-2001-x

15. Beffa LR, Margiotta AL, Carbonell AM. Flank and Lumbar Hernia Repair. Surg Clin North Am. 2018;1-13. DOI: 10.1016/j.suc.2018.01.009

16. Phillips MS, Krpata DM, Blatnik JA, et al. Retromuscular Preperitoneal Repair of Flank Hernias. J Gastrointest Surg. 2012;16(8):1548-53. DOI: 10.1007/s11605-012-1890-x

17. Moreno-Egea A, Moreno Latorre A. Actualización en la técnica de doble reparación protésica: experiencia personal y revisión de la literatura. 2019;7(4):144-50.

Tabla I. Comorbilidades

\begin{tabular}{|l|l|}
\hline Comorbilidades & Pacientes (n) \\
\hline Hipertensión Arterial & 16 \\
\hline Diabetes mellitus de tipo 2 & 8 \\
\hline $\begin{array}{l}\text { Enfermedad pulmonar obstructiva } \\
\text { crónica }\end{array}$ & 1 \\
\hline Enfermedad renal crónica & 1 \\
\hline Enfermedad coronaria & 1 \\
\hline Hipotiroidismo & 8 \\
\hline Malignidad & 3 \\
\hline
\end{tabular}


Tabla II. Características quirúrgicas

\begin{tabular}{|l|l|}
\hline Características & Pacientes (n) \\
\hline Antecedente quirúrgico & \\
\hline Apendicectomía & 8 \\
\hline Colecistectomía & 6 \\
\hline Cierre estoma & 6 \\
\hline Nefrectomías & 7 \\
\hline Reparación de aneurisma aorta & 0 \\
\hline Localización & \\
\hline L1 & 6 \\
\hline L2 & 13 \\
\hline L3 & 8 \\
\hline L4 & 0 \\
\hline Tamaño & \\
\hline W1 & 5 \\
\hline W2 & 14 \\
\hline W3 & 8 \\
\hline Técnica quirúrgica & \\
\hline Premuscular & 14 \\
\hline Retromuscular & 7 \\
\hline Laparoscópica & 3 \\
\hline Cierre primario abierto & 3 \\
\hline Tipo de malla & \\
\hline Polipropileno & 22 \\
\hline Compuestas & 1 \\
\hline Biológica & 1 \\
\hline Sin malla & 3 \\
\hline Recidiva & 3 \\
\hline Complicaciones & \\
Dindo) & \\
\hline I & 2 \\
\hline II & 3 \\
\hline III & 1 \\
\hline IV & 0 \\
\hline V & 0 \\
\hline & \\
\hline
\end{tabular}

